\title{
Feature Extraction and Recognition of Analog Circuit Fault Signal Based on Wavelet Transform and Partial Singular Values Decomposition
}

\author{
Liang Si-yang \\ Beijing Insititute of Technology, \\ Beijing 10081, P.R.China
}

\begin{abstract}
A new algorithm for the effective characteristics' extraction of analog circuit fault signal is proposed with consideration of its complexity. The proposed method is based on wavelet transform and partial singular values decomposition. Firstly, the analog circuit fault signal is decomposed into different scales and construct the initial feature matrix with wavelet coefficients, then divide the matrix into different sub-matrix, calculate the largest singular value of each sub-matrix, and the eigenvectors are constructed by the largest singular value. Then the support vector machine (SVM) with the constructed eigenvectors as its input eigenvectors is employed to recognize the analog circuit signal of different fault mode. The results show that the characteristics of analog circuit signal are efficiently extracted and the recognition precision of SVM is also improved, the new algorithm is a feasible way to process analog circuit fault signal.
\end{abstract}

Keywords-wavelet transform; singular value decomposition; feature extraction; analog circuit

\section{INTRODUCTION}

Analog circuit fault diagnosis has become a hot research area in modern circuit theory since the 1960s; it is the third branch of the network theory ${ }^{[1]}$. A variety of methods are used in analog circuit fault diagnosis by many experts and scholars. However, as the universality and complexity of the components parameters in the analog circuit, there are many problems that need to be solved. Wavelet transform has the character of good time-frequency trait and multi-division analysis, it has been used to extract feature and signal filtering, and it is an effective method for processing the analog circuit fault signal ${ }^{[2]}$.

M.Aminian treat the wavelet transform as preprocessor ${ }^{[3]}$, and extract the low frequency signal as characteristics of analog circuit fault by the wavelet transform, but the high-frequency part of the signal is discarded as noise, this may loss the effective ingredient of fault signal and influence the recognition accuracy of fault type.

The singular value decomposition is difficult influenced by disturbance, and it is a matrix algebra feature extraction method. A new algorithm of analog circuit fault signal feature extraction is proposed based on wavelet transform and partial singular values decomposition with consideration of the fault signal characteristics, the fault characteristics are extracted from the wavelet transform coefficient matrix.

\author{
Lv Jian-hong \\ Shijiazhuang Mechanical engineering college, \\ Hebei, Shijiazhuang, 050003, P. R. China
}

\section{WAVELET TRANSFORM AND SINGULAR VALUE DECOMPOSITION}

\section{A The wavelet transforms theory}

The wavelet transform is a time-scale analysis method; it can analyze the local signal characteristics in the domain of time-scale. The signal can be decomposed at different scales (from high to low frequency) signal components by wavelet transform, these signal components include the time information of the signal at a certain frequency. Temporal resolution is lower and frequency resolution is higher in the low-frequency part of the signal; while temporal resolution is higher and frequency resolution is lower in the high -frequency part of the signal.

The wavelet decomposition and reconstruction can be achieved by the Mallat algorithm. The decomposition formula is as follows.

$$
\left\{\begin{array}{l}
c_{k}^{j}=\sum_{l \in Z} c_{l}^{j+1} \bar{h}_{l-2 k} \\
\omega_{k}^{j}=\sum_{l \in Z} c_{l}^{j+1} \bar{g}_{l-2 k} \\
\bar{h}_{l-2 k}=\left\langle\varphi_{j+1, l}(x), \varphi_{j, k}(x)\right\rangle \\
\bar{g}_{l-2 k}=\left\langle\varphi_{j+1, l}(x), \phi_{j, k}(x)\right\rangle
\end{array}\right.
$$

Where $j, k \in Z, \varphi$ is the scaling function, $\phi$ is the wavelet function, $c_{k}^{j}$ is the $k$ low frequency coefficient when the scale is $j ; \omega_{k}^{j}$ is the $k$ high frequency coefficient when the scale is $j$.

Wavelet reconstruction algorithm is as follows.

$$
\left\{\begin{array}{l}
c_{k}^{j+1}=\sum_{l \in Z} c_{l}^{j} h_{k-2 l}+\sum_{l \in Z} \omega_{l}^{j} g_{k-2 l} \\
h_{k-2 l}=\left\langle\varphi_{j, l}(x), \varphi_{j+1, k}(x)\right\rangle \\
g_{k-2 l}=\left\langle\phi_{j, l}(x), \varphi_{j+1, k}(x)\right\rangle
\end{array}\right.
$$




\section{$B \quad$ The choice of wavelet basis}

The choice of wavelet basis is extremely important when process the signal by wavelet transforms. It is closely related to the specific application and it is the key of success. At present, there is no conclusive method about how to choose the wavelet basis. Daubechies wavelet, db2, db4, db6, biorthogonal wavelet is the most commonly used wavelet basis. The coefficients of low frequency and high frequency can be extracted by these different types of wavelet basis, and then achieve the feature extraction with the coefficients. db8 wavelet basis decomposition is used in this paper with consideration of the character of analog circuit fault signal.

\section{The singular value decomposition theory}

Based on singular value decomposition theory, the matrix $A_{a \times b} \in R^{a \times b}$ must exist two orthogonal matrices $U_{a \times b}$, $V_{a \times b}$ and one diagonal matrix $\Lambda$ that meet the following algorithm.

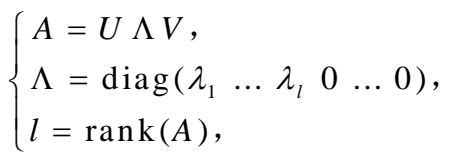

Where the $\lambda_{i}(i=1, \ldots, l)$ is the eigenvalue of matrix $A A^{\mathrm{T}}$.

As $\Lambda$ is a diagonal matrix, singular value decomposition is to divide the matrix $a \times b$ whose rank is $l$ into $l$ matrixes $a \times b$ whose rank is one, each matrix consists of two feature vectors (belonging to the matrix $U$ and the matrix $V$ ). The singular values can reflect the information of the matrix, and it is the characteristics of the matrix.

The stability theorem of the matrix singular value as follows: $A_{a \times b}, B_{a \times b} \in R^{a \times b}$, the singular value of the two matrix are $\lambda_{1} \geq \lambda_{2} \geq, \ldots, \lambda_{1}$ and $\delta_{1} \geq \delta_{2} \geq, \ldots, \delta_{l}$, then

$$
\left|\lambda_{i}-\delta_{i}\right| \leq\|A-B\|_{2} \quad i=1,2 \ldots l,
$$

Where $\|A-B\|_{2}$ is 2-norm of the matrix, its value is the square root of the matrix's largest singular value.

The time-frequency matrix of a signal is $A$,then the matrix becomes $B$ when the signal has been influenced by the interference. Expressions (4) shows that the change of singular value will under $\|A-B\|_{2}$. Therefore, treat the singular values of the time-frequency matrix as the matrix's parameters can reduce the variability between similar samples, and the singular values can reflect the characteristics of the time-frequency matrix effectively.

\section{FEATURE EXTRACTION}

Processing the analog circuit signal by wavelet transform with proper wavelet basis, then construct the wavelet transform matrix with the wavelet coefficients. The wavelet coefficients matrix is expressed as follows.

$$
M=\left[\begin{array}{l}
A_{i, n} \\
D_{i, n} \\
D_{i-1, n} \\
\cdot \\
\cdot \\
D_{1, n}
\end{array}\right]
$$

Where $\mathrm{A} i$ is the low frequency coefficients and $\mathrm{Di}$ is the high frequency coefficients, $i$ is the layer of wavelet transform, $(i=1,2,3 \ldots), \quad n$ is the number of wavelet coefficients. The layer of wavelet transform is 15 in this paper.

The matrix of wavelet coefficients contains the information of analog circuit fault. If calculating singular values of the entire matrix directly, the computation will be very large, and it is not advantaged for feature extraction. Besides, the singular value of the entire matrix does not include local characteristics, and contains a lot of redundant information ${ }^{[4]}$. Therefore, in order to get more information of the singular value, a new algorithm for the effective characteristics' extraction of analog circuit fault signal is proposed.

Singular value feature extraction method is improved in this paper, the wavelet coefficient matrix divided to four segments from the horizontal and vertical axis; there are 16 sub-matrixes in all. Then calculate the singular value of each sub-matrix, take the largest singular value of each sub-matrix composed the characteristics vector ( $T$ ) of the analog circuit, and $T=\left[\lambda_{11}, \lambda_{12}, \ldots, \lambda_{44}\right], \lambda_{i j}$ is the largest singular value of sub-matrix, $i$ is number of rows in matrix, $j$ is the number of columns in matrix, $i, j=1,2,3,4$.

\section{SUPPORT VECTOR MACHINE CLASSIFIER}

Support vector machine is more effective to solve the problems of small sample and nonlinear than the neural networks. Bayesian classifier and linear classifier, and it has the characteristic of higher computational efficiency ${ }^{[5]}$. Therefore, support vector machine is chosen as classifier in this paper.

$$
\left\{\left(x_{i}, y_{i}\right), i=1,2, \cdots, l\right\} \text { is the training sample set, }
$$
$y_{i} \in\{-1,1\}$ is the sample index set, $l$ is the number of samples, $x_{i} \in \mathrm{R}^{n}, n$ is the input dimension. Supposing $H: w \cdot x+b=0$ is the classification hyperplane of the sample set, it can divide the sample set into two types of samples. The $X_{i}$ which is the point of classification hyperplane meet the following algorithm.

$$
w \cdot x+b=0
$$

Where $w$ is normal vector of the hyperplane, $b$ is the 
offset.

Sample set is completely separated into two types as follows:

$$
\begin{cases}w \cdot x_{i}+b \geq 1 & y_{i}=1 \\ w \cdot x_{i}+b \leq-1 & y_{i}=-1\end{cases}
$$

The classification interval of classification hyperplane ( $H$ ) meet the following algorithm:

$$
M=\frac{2}{\sqrt{w^{T} w}}=\frac{2}{\|w\|}
$$

In order to reduce the error rate of classifier, the classification interval $M$ of hyperplane should be maximum. $\frac{1}{2}\|w\|^{2}$ is constructed to simplify the operation of the following quadratic programming problem.

$$
f(w)=\frac{1}{2}\|w\|^{2}
$$

As the optimal function is quadratic and the constraints are linear, the problem is a quadratic programming problem. Solving this problem by Lagrange factor as follows.

$$
\operatorname{Max} H(\alpha)=\sum_{i=1}^{l} \alpha_{i}-\frac{1}{2} \sum_{i, j=1}^{l} \alpha_{i} \alpha_{j} y_{i} y_{j}\left(x_{i} \cdot x_{j}\right)
$$

Where $\sum_{i=1}^{l} \alpha_{i} y_{i}=0 \quad \alpha_{i} \geq 0, i=1,2, \cdots, l$

$\alpha_{i}$ is the Lagrange factor. After getting the optimal hyperplane $\left(w^{*}, b^{*}\right)$, the sample $X$ are classified by the discriminant function as follows.

$$
f(X)=w^{*} \cdot X+b^{*}=\sum_{i=1}^{l} y_{i} \alpha_{i}^{*}\left(X \cdot x_{i}\right)+b^{*}
$$

$f(X)$ is the support vector machine.

If the sample is linearly inseparable, it can be translated into linearly separable of high-dimensional through a nonlinear transformation, then get the space optimal hyper plane in the high-dimensional.

\section{RECOGNITION OF ANALOG CIRCUIT FAULT BASED ON SUPPORT VECTOR MACHINE}

Diagnostic circuit is CTSV filter in this paper (as shown in Figure 1).

Where $R_{1}=R_{2}=R_{3}=R_{4}=R_{5}=10 \mathrm{k} \Omega, R_{6}=3 \mathrm{k} \Omega$ ， $R_{7}=7 \mathrm{k} \Omega, C_{1}=C_{2}=20 \mathrm{nF}$. There are four fault mode, respectively fault: $R_{1}+50 \%$ or $C_{1}-50 \%$; multiple faults: $R_{1}+50 \%$ and $C_{1}-50 \%$; and circuit work properly.

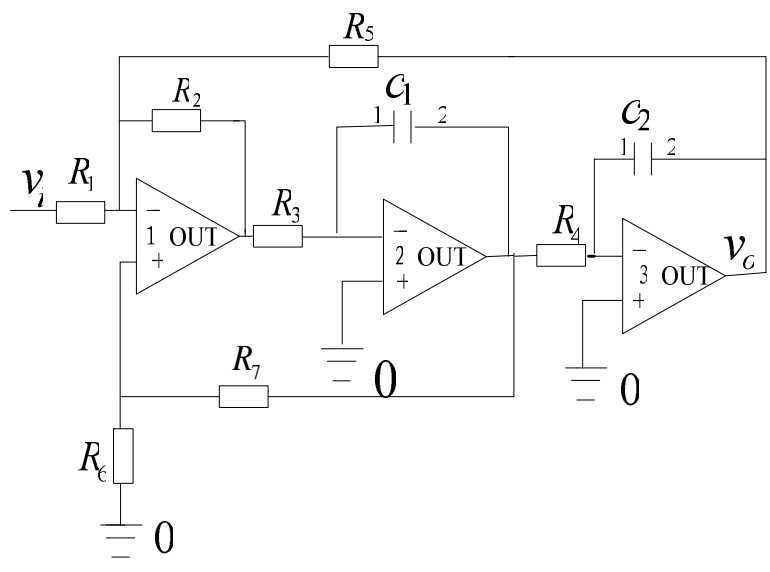

Figure 1 Continuous-time state-variable filter

The analog circuit faults are tested by simulation. Treating the eigenvector that is extracted by the proposed algorithm as optimized eigenvector, extracting the optimized eigenvector and the traditional eigenvector (the wavelet matrix is not divided). Then the support vector machines with optimized eigenvector and traditional eigenvector as its input eigenvectors is introduced to recognize the analog circuit fault signal. The number of eigenvector elements is 16 . There are 40 samples in each fault sample set. After the support vector machines are trained, the samples of each fault mode are tested by support vector machines the test results are shown in Table 1.

Table 1. THE RESULT OF SUPPORT VECTOR MACHINES WITH THE DIFFERENT EIGENVECTOR AS ITS INPUT EIGENVECTORS

\begin{tabular}{rcc}
\hline \hline eigenvector & optimized eigenvector & traditional eigenvector \\
\hline veracity of & & \\
recognition & $87.5 \%$ & $80 \%$ \\
\hline \hline
\end{tabular}

It can be seen from Table 1, the veracity of support vector machines is improved effectively and the proposed approach is a feasible feature extraction algorithm for analog circuit fault signal.

\section{CONCLUSION}

A new algorithm for the effective characteristics' extraction of analog circuit fault signal is proposed based on wavelet transform and partial singular values decomposition. The matrix is constructed by wavelet coefficients of analog circuit fault signal, and then the wavelet coefficient matrix is divided into different sub-matrix from the horizontal and vertical axis considering of the signal's complexity, the optimized eigenvector is extracted from this sub-matrix. The performance of the proposed approach is verified by simulation. The result indicates that the new algorithm can extract the feature of analog circuit fault signal, and improve the recognition veracity of support vector machines 


\section{effectively.}

\section{REFERENCES}

[1] Xiao Yingqun, He Yigan. A fault diagnosis approach for analog circuits based on wavelet fractal analysis and ridgelet network[J].Transactions of China Electro Technical Society,2011,26(11):105-114

[2] Yi Fang, Li Zhuxin, Su Yi, et al. De-noising algorithm for metal magnetic memory signals of oil pipeline based on improved wavelet threshold[J].Acta Petrolei Sinica , 2009, 30(1): 141-144.
[3] He Yigang, Zhu Wenji, Zhou Yantao, et al. An analog circuit diagnosis method based on particle swarm optimization algorithm[J]. Transactions of China Electro Technical Society,2010,25(6):163-171

[4] Du Lin, Dai Bin, Lu Guojun, et al. Overvoltage features extraction based on $\mathrm{S}$ transform and local singular value decomposition[J]. Transactions of China Electro Technical Society,2010,25(12):147-153

[5] Wang Yanjing, Qiao Xiaoyan, Li Peng, et al. Classification of motor imagery task based on wavelet packet entropy and support vevtor machines[J]. Chinese Journal of Scientific Instrument, 2010, 31(12): $2729-2735$ 